\title{
Ein Beitrag zur Morphologie, Biologie und systematischen Stellung des Polychaeten Ctenodrilus serratus
}

\author{
M. WILFERT \\ Institut für Zoologie der Universitüt Düsseldorf; \\ Düsseldorf, Bundesrepublik Deutschland
}

\begin{abstract}
A contribution to the morphology, biology and systematic position of the polychaete Ctenodrilus serratus. The drilomorph polychaete Ctenodrilus serratus (O. SсHмDT 1857 ) is described. The following features are variable: size, colour of epidermal glands, number of bristles within one bundle, shape of bristles. According to these criteria C. s. limulicolus is not a valid subspecies. The pharyngeal bulb (buccal bulb) of the ctenodrilids can be compared with that of the archiannelids; it cannot be homologized with the buccal bulb of the genus Potamodrilus (Oligochaeta). This pharyngeal bulb is important for feeding and movement. C. serratus and the other members of the family Ctenodrilidae have a ventral ciliary field on the first segments, which has apparently no function for feeding and locomotion. The presence of this field and the epidermal glands, as well as the absence of paired tentacles and gills enable the Ctenodrilidae to be separated from the Cirratulidae.
\end{abstract}

\section{EINLEITUNG}

Die erste, jedoch noch recht unzureichende Beschreibung der Polychaetengattung Ctenodrilus findet sich bei O. SсHмтDт (1857); er nannte die von ihm untersuchte Form Parthenope serrata. Claparède (1863) konnte genauere Angaben über diese Art machen; von ihm stammt auch der Gattungsname Ctenodrilus. Schmots und Claparèdes Bezeichnungen wurden zu dem heute gültigen Namen Ctenodrilus serratus vereinigt (endgültig von Monticelli 1893). Sudzuki \& SEKIguchi (1972) stellten vor kurzem eine neue Unterart als C. s. limulicolus vor.

SCHARFF (1887) beschrieb als C. parvulus eine weitere Art, die sich von C. serratus durch die geringere Größe und die andersartige Ausbildung der Borsten unterscheidet.

Weitere Untersuchungen an den Ctenodriliden befaßten sich hauptsächlich mit ihrer systematischen Stellung (Vejoowrsky 1884, Caullery \& Mesnil 1898), der Anatomie und Histologie (KenNEL 1882, Galvagnd 1903) sowie der Fortpflanzungsbiologie (Kennel 1882, Monticelli 1906, Sokolow 1911).

C. serratus-Funde aus Jugoslawien und einem Meerwasser-Aquarium boten nunmehr die Möglichkeit, einige Widersprüiche in den Angaben der verschiedenen Autoren zu klären und weitere ergänzende Beobachtungen zur Kenntnis dieser Polychaeten- 
Gattung zu machen. Besonders berücksichtigt wurden dabei der Pharynxbulbus und das ventrale Wimperfeld auf den vordersten Borstensegmenten; den phylogenetischen Wert beider Organe im Hinblidk auf die Verwandtschaftsbeziehungen zwischen Anneliden und Mollusken hat erst kürzlich SALVINI-PLAWEN (1972) erörtert.

$\mathrm{Da}$ über die Lebensweise von $\mathrm{C}$. serratus bisher kaum Einzelheiten bekannt waren (vgl. HARTMANN-SCHRÖDER 1971), werden auch einige biologische Daten mitgeteilt.

\section{MATERIAL UND METHODEN}

Herkunft: 3 verschiedene Stämme (A-C) von Ctenodrilus serratus dienten als Untersuchungsmaterial. Stamm A wurde in Kulturschalen gefunden, in denen im Institut für Zoologie der Universität Düsseldorf ein marines Kamptozoon (Barentsia spec.) aus Palo Alto (Kalifornien) gezüchtet wurde. Die Tiere des Stammes B wurden in Rovinj (Istrien, Jugoslawien) zwischen Kalkrotalgen (Corallina mediterranea) gefunden. Ebenfalls aus Rovinj kam Stamm C; diese Tiere lebten zwischen Detritus in der Uferzone.

Die Zucht erfolgte bei Zimmertemperatur in Petrischalen von $20 \mathrm{~cm} \phi$ in Seewasser, dem ein sterilisiertes, leicht gequetschtes Weizenkorn zugegeben war. Etwa 1-2mal pro Woche wurden Grünalgen der Gattung Dunaliella hinzugefügt.

Untersuchungstechnik: Lebende Tiere wurden mit einem Roto-Kompressor (WrLFERT \& BECKMANN 1972) festgelegt und beobachtet; zusätzlich (und auch vor jeder Fixierung) wurden sie mit 7 prozentiger $\mathrm{MgCl}_{2}$-Lösung oder mit Chloreton $(0,5 \%)$ betäubt. Im Dunkelfeld sind an lebenden Tieren besonders gut Cilien und Tasthärchen zu erkennen.

Die Fixierung für die Lichtmikroskopie erfolgte mit Bouin-Duboscq-Brasil, gefärbt wurde mit Dobell-Chromotrop oder Azan (Einbettung in Paraffin; Schnittdicke: $3 \mu \mathrm{m})$. Nach der Methode von PETERS (1961) wurden Aufhellungspräparate angefertigt, die mit Alaunkarmin oder Direkttiefschwarz angefärbt wurden.

Präparationsweg für die Raster-Elektronenmikroskopie, speziell für Cilien-Darstellung: Fixierung in einem $1: 1-$ Gemisch von 2prozentiger Osmiumsäure und Meerwasser; Auswaschen in Alkohol (70-100\%); anschließend Karbol : Xylol $1: 1$ (90 min), Xylol : Terpentinöl $2: 1$ (90 min), gereinigtes Terpentinöl (90 min). Bedampfung mit Gold, Untersuchung im JEOL Scanning Electron Microscope ISM-U3.

\section{MORPHOLOGISCHE DATEN}

\section{Außere Organisation}

Ctenodrilus serratus ist ein sehr kleiner Polychaet (Abb. 1). Einzeltiere erreichen Längen von 1,1-2,5 mm (Stamm A), 1,2-2,8 mm (Stamm B) und 1,2-2,4 mm (Stamm C). Die Körperbreite beträgt je nach Kontraktionszustand 90-120 $\mu \mathrm{m}$.

C. serratus bildet durch Paratomie Tierketten, die 3-5 mm (A,C) und 4-7 $\mathrm{mm}$ (B) lang sind. Sie können aus bis zu 6 (A, C) oder 7 (B) Einzeltieren bestehen; vorhert- 
schend sind Ketten aus 4-5 Zooiden. Die Lage der ersten Teilungszone ist recht konstant, sie liegt zwischen dem 3. und 4. oder 4. und 5. borstentragenden Segment.

Ein charakteristisches Aussehen erhält C. serratus durch farbige Hautdrüsen (Abb. 1, 2). Sie sind braun (Stämme A, C) oder blau bis schwarzviolett (B), 4-8 $\mu \mathrm{m}$ groß und in den meisten Fällen dicht und gleichmäßig über den ganzen Körper verteilt. Intensität und Grad der Pigmentierung können jedoch stark schwanken. Die Drüsenzellen sind unregelmäßig gestaltet; das Sekret in ihnen liegt in kugelförmigen Grana vor.

Am ganzen Körper finden sich härchenartige Fortsätze ("Tasthärchen“ in der Polychaeten-Literatur). Sie treten gehäut am Vorderrand des Prostomiums und am Hinterende des Tieres auf (Abb. 1); von älteren Autoren wurden sie nicht beschrieben.

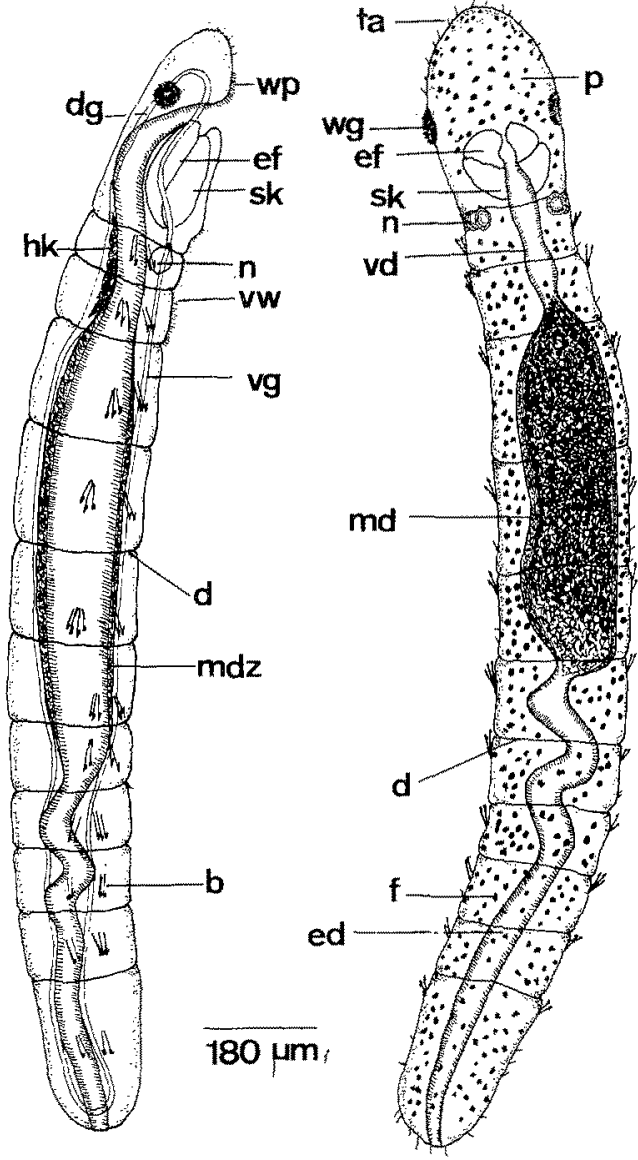

Abb. 1: Ctenodrilus serratus; links: Seitenansicht (ohne Segmentalgefäße und Hautdrüsen); rechts: Dorsalansicht (ohne Gefäßsystem). b Borste, $d$ Dissepiment, $d g$ Dorsalgefäß, ed Enddarm, ef Falte des Pharynxtaschen-Epithels, $f$ farbige Hautdrüse, bh Herzkörper, md Mittel$\mathrm{darm}, m d z$ braungefärbte Mitteldarmzellen, $n$ Nephridium, $p$ Prostomium, sk Schlundkopf, ta Tasthärchen, wd Vorderdarm, ww ventrales Wimperfeld, wg Nuchalorgan, wp Wimperfeld auf dem Prostomium 
Das Prostomium trägt an den Seiten je ein Nuchalorgan, ein rundes Wimperfeld mit einem Durchmesser von $19 \pm 3 \mu \mathrm{m}$ (Abb. 1,3). Ventral ist das Prostomium ausgehöhlt; hier ist es mit Cilien ausgekleidet, an die sich kontinuierlich die Wimpern der weiten Mundhöhle anschließen.

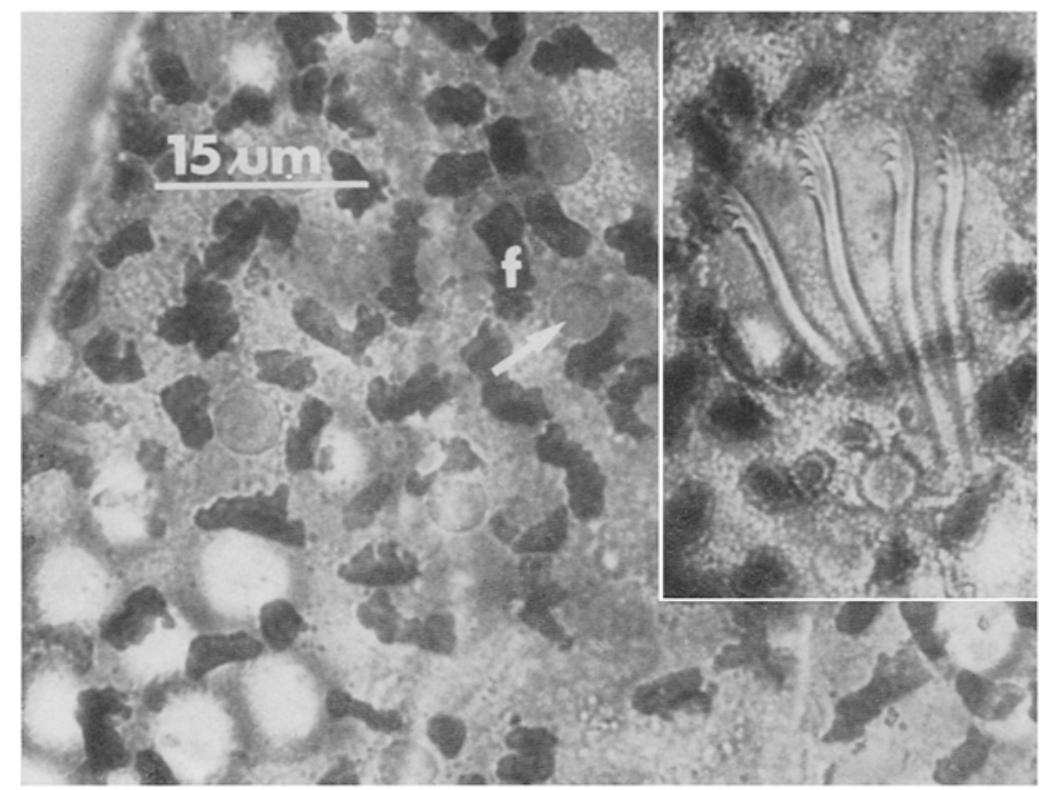

Abb. 2: Farbige Hautdrüsen (f) von Ctenodrilus serratus; der Pfeil weist auf eine Cölomozyte. Oben rechts: Borstenbündel mit den für Ctenodrilus serratus charakteristischen gezähnten Borsten

Hinter dem Prostomium folgt das borstenfreie Schlundkopf-Segment (p. 337 und Abb. 1), in dem ein weiteres Wimperfeld beginnt, dessen Umfang bei früheren Untersuchungen (KENNEL 1882, GALVAGNT 1903) nur unzureichend bekannt wurde (Abb. 4). Es ist in Form eines langgezogenen gleichschenkligen Dreiecks ausgebildet (Länge: $200-250 \mu \mathrm{m})$. Die Grundseite dieses Dreiecks beginnt im Schlundkopfsegment, seine Spitze liegt am Ende des 2. Borstensegments.

Die Parapodien von $C$. serratus sind völlig bis auf die charakteristische Beborstung geschwunden. Die Borsten stecken ventrolateral an jeder Körperseite in Taschen und sind in je 2 Bündeln angeordnet; dies gilt grundsätzlich für alle untersuchten Stämme und klärt unterschiedliche Beobachtungen älterer Untersuchungen (ScHMIDT 1857, Claparède 1863, KenNel 1882, Vejdowsky 1884). Tiere zwischen 1 und 2 mm Körperlänge besitzen 9-12 (meist 10-11) Borstensegmente.

Die Anzahl der Borsten innerhalb eines Bündels auf einer Körperseite schwankt $z$ wischen 1 und 5 , die meisten Borsten finden sich in den mittleren Segmenten. Die Borsten sind im Vorder- und Enddarm-Bereich etwa $45 \pm 8 \mu \mathrm{m}$ lang, im Mitteldarm-Abschnitt sind sie mit $70 \pm 5 \mu \mathrm{m}$ etwas länger. Sie bestehen aus einem annähernd geraden 
Schaft, der sich im letzten Teil leicht krümmt. Der gebogene Teil trägt auf der Konkavseite 4-7 unterschiedlich geformte Zähnchen (Abb. 3). Untersuchungen mit dem REM zeigten, daß gelegentlich auch die Konvexseite zähnchenartige Fortsätze tragen kann.

Der Chitosantest (vgl. Peters 1968) an den Borsten verlief negativ; da sie in KOH gelöst wurden, ist $\mathrm{zu}$ vermuten, daß sie kaum Chitin enthalten und im wesentlichen aus Protein bestehen.

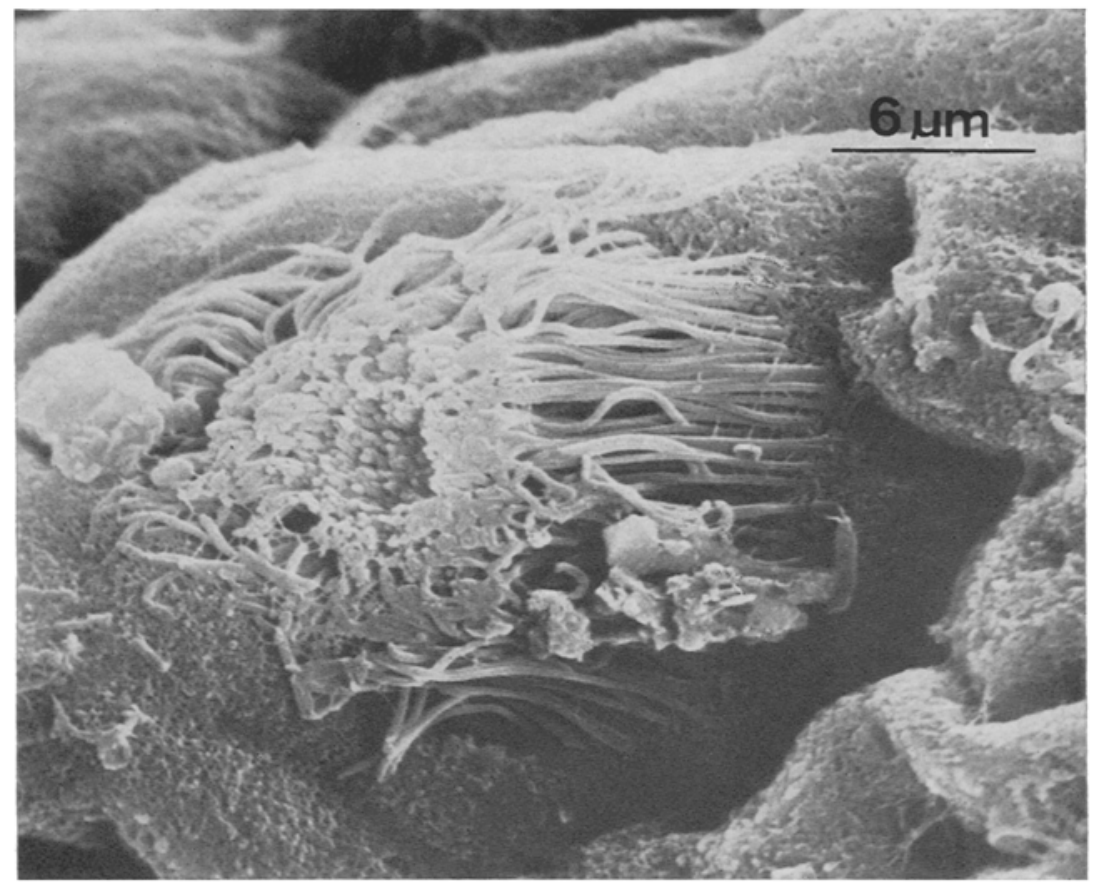

Abb. 3: Nuchalorgan von Ctenodrilus serratus; Ansicht von schräg hinten

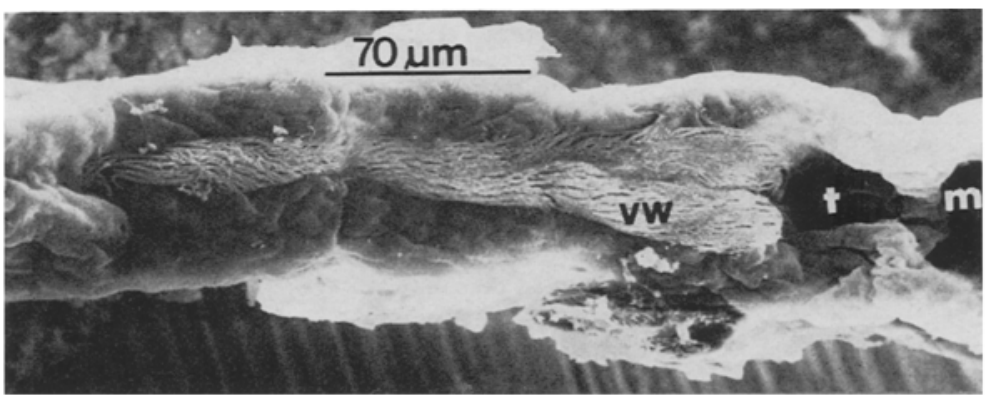

Abb. 4: Aufsicht auf das ventrale Wimperfeld (vw); $t$ Pharynxtasche, $m$ Mundhöhlenbereich 


\section{Innere Organisation}

Auffallend ist das Fehlen von Geschlechtsorganen. Auch bei den mehr als 5 Jahre in Kultur gehaltenen Tieren des Stammes A traten niemals Gonaden auf; die Fortpflanzung erfolgte unter den angegebenen Kulturbedingungen ausschließlich durch Paratomie.

Der Darmkanal kann in leichten Schlingen liegen; er ist durchgehend bewimpert. Im Prostomium macht der Vorderdarm eine Biegung nach ventral und öffnet sich durch den weiten Mund nach außen. An diese Mundhöhle schließt sich caudad eine Pharynxtasche an (Abb. 1,4), deren Höhlung durch Epithelfalten und einen ausstülpbaren. Pharynxbulbus eingeengt wird (Abb. 1). Der Aufbau von Pharynxtasche, Pharynxbulbus und der Verlauf der Muskulatur wurden weder von KENNEL (1882) und GALVAGNI (1903) noch von SoKolow (1911) insgesamt richtig dargestellt.

Das aus großen Zellen bestehende bewimperte Vorderdarmepithel (Abb, 5a, b) der Mundhöhle biegt an der Mundöffnung caudad um und bildet ein wimperloses Pharynxtaschen-Epithel, das aus wesentlich kleineren Zellen besteht. Etwa in Höhe des Umbiegens des Vorderdarms nach ventral macht auch dieses Epithel eine Biegung nach proximal und bildet dadurch links und rechts von der Medianebene zwei große Falten, die halbkreisförmig in die Pharynxtasche hineinragen (Abb. 1; 5a, c). Die beiden Falten sind hervorstreckbar (s. unten) und können, wenn sie in der Medianebene aneinanderstoßen, die Mundöffnung weitgehend verschließen.

Vom distalen Ende der Falte ausgehend verläuft das Pharynxtaschen-Epithel wieder nach vorn (Abb. 5a), parallel zum Vorderdarm und bildet so die ventrale Auskleidung der Pharynxtasche, bis es schließlich auf die ventrale Epidermis triff ( $A b b .5 a$ ).

Dem Epithel unterhalb der Falte liegt ein unpaares Muskelpolster an („Unterlippe“ bei Kennel 1882, "Schlundkopf“ bei Galvagni 1903). Dieser Schlundkopf (Pharynxbulbus; Abb. 5a, c, d,6) hat etwa die Form einer nach hinten zu größer werdenden Schaufel: In der Aufsicht gesehen sind die beiden Seitenränder emporgewölbt und laufen proximal in zwei abgerundete Zipfel aus, die durch eine Einbuchtung miteinander verbunden sind (Abb.6). Das distale Ende des Schlundkopfes ist halbkreisförmig gerundet.

Kennzeichnend für den Bau des in sich abgeschlossenen Schlundkopfes sind zur Körperlängsachse quergestellte, lamellenartig hintereinander angeordnete Muskelplatten (Abb. 5a,6). Jede dieser Platten besteht aus zwei Einzelplatten, die dicht aneinanderliegen und kurz vor der Insertion am Epithel des Bulbus sich in ihre beiden Anteile aufspalten (Abb. 5a). GALVAGN (1903) fand im Schlundkopf auf mit Eisenhämatoxylin gefärbten Schnitten "Stützfibrillen“, die sich bei der Untersuchung mit Azan nicht darstellen ließen. Auch Sokolow (1911) konnte bei Raphidrilus keine derartigen Fasern nachweisen.

An der paarigen großen Epithelfalte sowie am ventralen Epithel des Vorderdarms greifen jeweils mehrere Muskelfaserzüge an, die zunächst weitgehend getrennt caudalwärts in Längsrichtung des Tieres verlaufen und sich kurz vor dem distalen Schlundkopf-Ende zu einem einheitlichen breiten Muskelstrang aneinanderlegen (Abb. 5a).

Dieser "Sagittalmuskel" legt sich nun an das Bulbusende an und umhüllt ihn ventral und an den Seiten, indem er im Bogen nach vorn biegt (Abb. 5a, c, d). An der 
Spitze des Schlundkopfes inseriert er in einzelnen Fasern an der ventralen Pharynxtaschenwand (Abb. 5a). Hier liegt auch die Anheftungsstelle für ein weiteres System von Muskelfasern, die unterhalb des Schlundkopfes parallel zu diesem laufen und kurz vor dem Dissepiment im Hautmuskelschlauch enden (Abb. 5a). Thre Kontraktion bereitet das Ausstullpen der Epithelfalten und des Pharynxbulbus vor. An ihrer Insertionsfläche wird das Pharynxtaschenepithel rück-und ventralwärts gezogen und bildet so eine Einbuchtung bzw. Falte (Abb. 5c). Durch Kontraktion des Sagittalmuskels werden die Epithelfalten und der Bulbus in proximale Richtung gedruickt; der Apex des

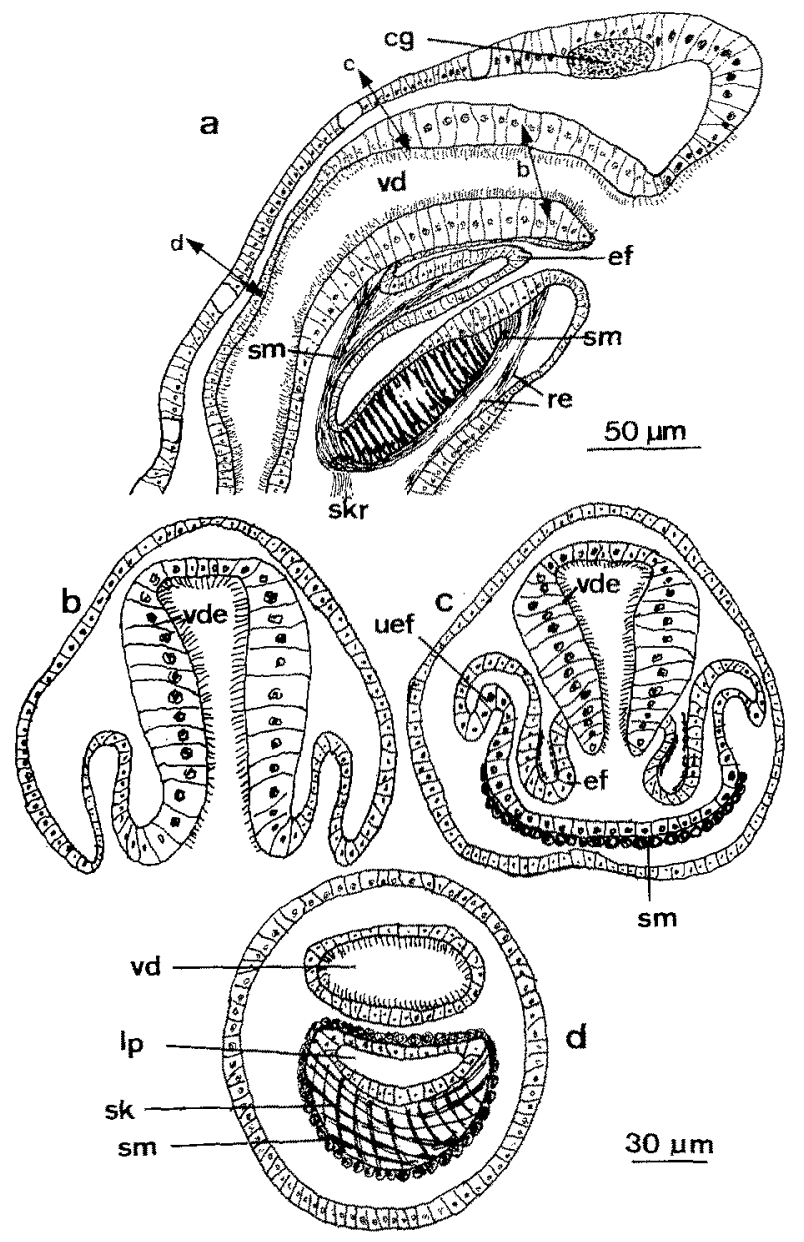

Abb. 5: Sagittal- und Querschnitte durch den Vorderkörper von Ctenodrilus serratus (schematische Ansichten). Im Sagittalschnitt a geben die Pfeile $b, c$ und $d$ die ungefähren Schnittrichtungen der Abbildungen $\mathrm{b}-\mathrm{d}$ an. $\mathrm{cg}$ Cerebralganglion, ef Falte des Pharynxtaschen-Epithels, Ip Lumen der Pharynxtasche, re Retraktor des Pharynxtaschen-Epithels, sm Sagittalmuskel, sk Schlundkopf, skr Schlundkopf-Retraktor, uef untere Epithel-Falte, vd Vorderdarm, vde Vorderdarm-Epithel 
Schlundkopfes wird dabei nach ventral umgeklappt. Er gleitet über das zurückgezogene Pharynxtaschenepithel, seine vordere Dorsalfläche liegt nunmehr dem Substrat an.

$\mathrm{Zu}$ diesen beiden Muskelsystemen treten zwei Retraktoren des Schlundkopfes. Das distale Bulbusende trägt in der Medianebene in Form großer blasiger Zellen besonders gut entwickeltes Cölomepithel (Abb.6). Links und rechts davon greifen am Schlund-

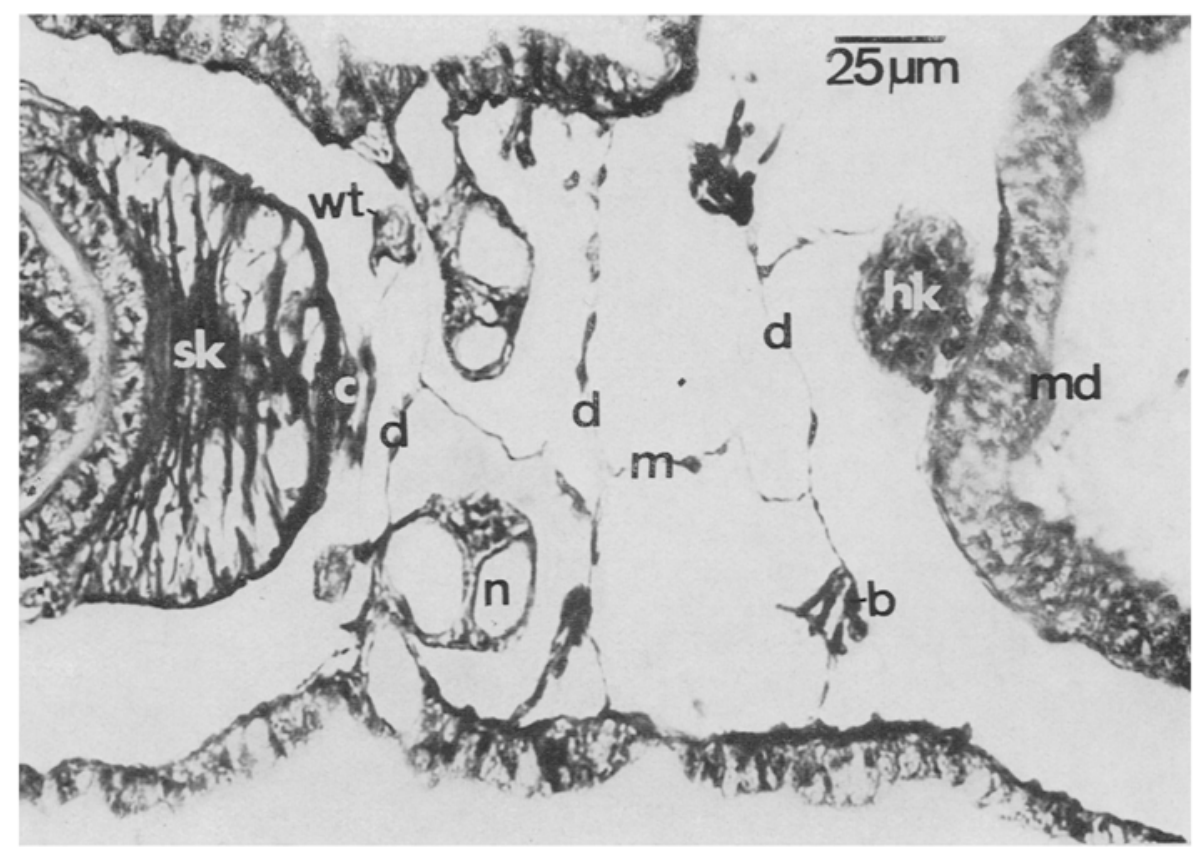

Abb. 6: Horizontalshnitt durch die ersten Segmente von Ctenodrilus serratus. $b$ Borste, $c$ verdicktes Cölomepithel, $d$ Dissepiment, $b k$ Herzkörper, $m$ Mesenterium, $m d$ Mitteldarm, $n$ Nephridialkanal (im oberen Nephridium ist der Nephridioporus angeschnitten), sk Schlundkopf, wt Wimpertrichter des Nephridiums

kopf zwei Rückziehmuskeln an (Abb. 5a), die sich parallel zum Darm caudalwärts fortsetzen und schließlich am Ventralepithel inserieren.

Als Haltemuskulatur des Bulbus, die nicht in den Vorgang der Pro- und Retraktion eingreift, sind feine Muskelfasern anzusprechen, die jeweils zwischen proximalem und distalem Schlundkopfende und der lateralen Epidermis ausgespannt sind.

An den Vorderdarm (Pharynx und Osophagus) schließt sich der stets breitere Mitteldarm an (Abb. 1), dessen Epithelzellen intensiy braun gefärbte Granula enthalten. Die Erweiterung zum Mitteldarm liegt ausnahmslos zwischen dem 2. und 3. Borstensegment; der Ubergang zwischen Mittel- und schmalerem Enddarm befindet sich meist zwischen dem 4. und 5. Borstensegment.

Auf Schnitten ließen sich im Darm keine peritrophischen Membranen nachweisen, wie sie Peters (1968) und Vrerhaus (1971) bei zahlreichen Anneliden gefunden hatten.

Das Blutgefäßsystem (Abb. 1) ist geschlossen und besteht aus einem Dorsal- und Ventralgefäß, die durch segmentale Ringgefäße verbunden sind. Das Dorsalgefäß spal- 
tet sich im Prostomium in zwei Anteile, die sich unterhalb des Darmes im Bulbus-Segment zu einem einheitlichen Ventralgefäß vereinigen. Kurz vor dem After werden beide Hauptstämme ebenfalls durch zwei kurze Seitenäste miteinander verbunden; GALVAGNI (1903) konnte diese Verbindung nicht beobachten, vermutete aber richtig, daß das Gefäßsystem vollkommen geschlossen ist.

Das Dorsalgefäß schließt in seinem ventralen Teil über dem Vorderdarm bis zum Mitteldarm-Beginn eine mehrschichtige Lage meist intensiv braungelb gefärbter Zellen ein (Abb. 1, 7), die durch Bindegewebsfasern zusammengehalten werden. Dieser „Herz-

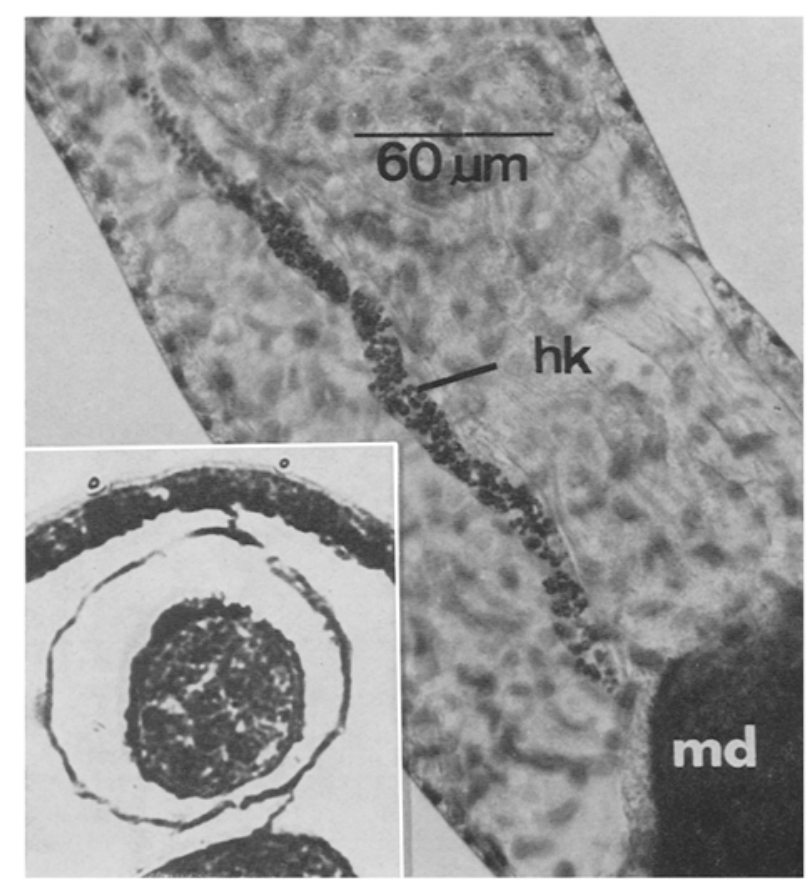

Abb. 7: Herzkörper ( $h k$ ) im Dorsalgefäß von Ctenodrilus serratus. Unten links: Querschnitt durca das Dorsalgefäß mit Herzkörper

körper", der auch bei anderen sedentären Polychaeten sowie einigen Oligochaeten zu finden ist (vgl. Stephenson 1930), hat eine noch unbekannte Funktion. Bei der Kontraktion des Gefäßes wird ex gegen die dorsale Herzwand gedrückt und übt so möglicherweise eine Ventilfunktion aus. Seine Primärfunktion könnte nach FAUvel (1959) die eines Stoffwechselorgans sein, vergleichbar eventuell einem Chloragog; dafür spräche bei Ctenodrilus, daß Farbe und Farbintensität der Herzkörperzellen sich ändern können.

C. serratus besitzt ein Paar Metanephridien (Abb. 1); ihr Wimpertrichter liegt im Segment des Schlundkopfes dem Dissepiment an (Abb. 6). Der Nephridialkanal durchbricht das Dissepiment, bildet mehrere Schleifen und mündet im ersten Rumpfsegment nach außen. Sudzuki \& Sekiguchi (1972) deuteten die Nephridien fälschlich als Speicheldrüsen. 
Das Nervensystem liegt, wie schon KeNNEL (1882) erkannte, völlig im Ektoderm verborgen. Die vom Cerebralganglion ausgehenden Konnektive treffen sich unterhalb des Schlundkopfes und bilden von dort ab das Bauchmark.

Schlundkopf-Segment und alle weiteren borstentragenden Segmente sind voneinander durch wohlausgebildete Dissepimente getrennt (Abb.6), auch die Mesenterien sind vollständig vorhanden (von GALVAGNI 1903 nidht erkannt). Zwischen den Dissepimenten des Hinterkörpers fallen frei flottierende Cölomzellen auf, deren Funktion noch nicht bekannt ist (Abb. 2).

\section{BIOLOGISCHE DATEN}

Die blaue Form von Ctenodrilus serratus konnte in großer Menge zwischen Corallina mediterranea gefunden werden, die in Rovin; (Jugoslawien) oft langausgedehnten Bewuchs auf Felsen der Uferzone bildet.

Die Bewegungen der meisr gekrümmten Tiere bestehen aus einem langsamen Vorwärtskriechen, ausgelöst durch peristaltische Bewegungen des Hautmuskelschlauchs; die Würmer bleiben in ständigem Kontakt mit dem Substrat. Zu Beginn der Vorwärtsbewegung erfolgt ein Ausstïlpen des Pharynxbulbus, der sich als Stützorgan gegen den Untergrund stemmt. Keine wesentliche Rolle bei der Bewegung spielen die Borsten; nur bei einem seitlichen Aufstützen des Körpers können sie als Widerlager oder Verankerung dienen. Auch die ventrale Bewimperung hat keinerlei Bedeutung für die Lokomotion.

Die Nahrung der Tiere besteht in erster Linie aus Detritus, aber auch "sperrige" Algen, wie z. B. Licmophora (Diatomeae), fanden sich im Darm. Beim Ausstülpen des Schlundkopfes schabt dieser Detritusmaterial von der Unterlage ab bzw. wirbelt es auf, das durch Einziehen des Bulbus sowie durch das Schlagen der Wimpern von Prostomium und Vorderdarm in den Mund befördert wird. Auch bei der Nahrungsaufnahme läßt sich dem ventralen Cilienfeld keine Funktion zuordnen, da seine Wimpern nur vom Mund weg caudalwärts schlagen.

In der Kulturschale bildeten die Würmer in einigen Fällen feine Röhren aus Hautsekret und Fremdstoffen; im natürlichen Biotop konnten derartige Schleimröhren nicht gefunden werden. Hier lebt $C$. serratus oft völlig versteckt zwischen Algenfäden und Detritus (was vor allem für die bratıngefärbten Tiere des Stammes $C$ zutraf); in solchen Fällen kann die Ventralbewimperung vielleicht der Erzeugung eines Atemwasserstroms und dem kontinuierlichen Abtransport der Exkretstoffe dienen.

Die in Kultur genommenen Tiere zeigten eine positive Phototaxis; stets wird die dem Licht zugewandte Seite der Petrischale bevorzugt.

\section{DISKUSSION}

Der Fund von. Ctenodrilus serratus in Rovinj (Jugoslawien) stellt einen Erstnachweis für die Fauna dieser Gegend dar. Katzmann (1972) erwähnte in seiner faunistischen Studie der Polychaeten Rovinjs von den Ctenodriliden lediglich die Gattung Raphidrilus. 
Der Vergleich der in Zucht genommenen Stämme zeigt die Variationsbreite der Art C. serratus auf: (1) Die Größe der Zooide schwankt zwischen 1 und 2,8 $\mathrm{mm}$. Die Angaben von 8-9 mm Länge bei FAuvel (1927) und HARTMANN-SCHRöder (1971) beziehen sich ohne Zweifel auf Tierketten aus mehreren Zooiden; Einzeltiere erreichen niemals derartige Körpergrößen. (2) Die Farbe der Hautdrüsen ist nicht konstant; zu den hier beobachteten Farben Blau bis Violett und dem erstmals nachgewiesenen Braun finden sich weitere wie Schwarz (Claparède 1863, Fauvel 1927, HartmanN-SchröDer 1971) und Grün (Kennel 1882, Galvagni 1903). Daß die Farbe innerhalb eines Stammes variabel sein kann, zeigte sich bei der Haltung von Stamm B, bei dem einzelne Tiere neben blauschwarzen auch gelbbraune Farbdrüsen aufwiesen. (3) Daß die Zahl der Borsten in einem Bündel unterschiedlich ist, bestätigen die Zählungen von KENNEL (1882); bei beiden Untersuchungen enthielten die mittleren Segmente die meisten Borsten. Somit können Zahl und Verteilung der Borsten nicht als artspezifisches Merkmal gelten.

Auf Grund dieser Merkmalsvariationen kann die von Sudzukr \& Sekiguch (1972) aufgestellte Unterart C. s. limulicolus nicht als valide betrachtet werden. Die Unterschiede gegenüber der Nominatform (Borstenanordnung, Borstenform, Körperlänge) fallen alle unter die Variationsbreite dieser Merkmale innerhalb eines Stammes von C. serratus.

Bei fast allen untersuchten Formen von C. serratus wurde als alleinige Fortpllanzung eine Vermehrung durch Teilung unter Bildung von Tierketten festgestellt. Der Ausdruck „Ctenodrilisation“, der für diesen Modus gebraucht wurde (DEHORNe 1924, FAUVEL 1959), erscheint überflüssig; es handelt sich um eine echte Paratomie (bei den Gattungen Zeppelina und Raphidrilus dagegen tritt Architomie auf). Lediglich MonTICELLI (1906) konnte eine kurze Beschreibung der geschlechtlichen Fortpflanzung geben.

Seit den Untersuchungen von Mesnil \& CaulLery (1897) sowie Caullery \& MESNIL (1898) werden die Ctenodrilidae als drilomorphe Polychaeten in die nahe Verwandtschaft der Cirratuliden gestellt; diese Einreihung beruht vor allem auf Ahnlichkeiten in der Anatomie zwischen frühen Entwicklungsstadien des Cirratuliden Dodecaceria concharum und den 3 Ctenodriliden-Gattungen Ctenodrilus, Zeppelina und Raphidrilus; die geschlechtliche Fortpflanzung erfolgt bei allen genannten Gattungen nach dem gleichen Modus.

Die Untersuchungen an C. serratus und der Vergleich mit Zeppelina und Raphidrilus lassen es ratsam erscheinen, diese 3 Gattungen als eigene Familie Ctenodrilidae Kennel 1882 neben die Cirratulidae Carus $1863 \mathrm{zu}$ stellen (Fauvel 1927 billigte ihnen lediglich den Rang einer Unterfamilie $\mathrm{zu}$ ).

Den Ctenodriliden fehlen die paarigen Tentakel und die Kiemen der Cirratuliden; sie lassen sich fernerhin von ihnen durch zwei Merkmale abtrennen, die die aus recht heterogenen Merkmalen zusammengestellte Familiendiagnose von HARTMANN-SCHRöDER (1971) ergänzen: Integument mit über den ganzen Körper verteilten farbigen Drüsen; Prostomium und erste Rumpfsegmente ventral bewimpert* (entgegen den An-

* Für Raphidrilus nemasoma widersprechen sich in dieser Hinsicht die Angaben von MoNTiCelli (1910) und SoKolow (1911). 
gaben Fauvels 1927 und Montrcellis 1910 trägt auch das erste Borstensegment von C. parvulus Cilien; vgl. Scharfa 1887 und GaLvagni 1903).

Für das ventrale Cilienfeld auf den ersten Segmenten von C. serratus ließ sich eine Funktion weder für die Fortbewegung noch für die Nahrungsaufnahme feststellen. Ahnliche Bildungen sind bei verschiedenen Archianneliden und Polychaeten-Larven bekannt (vgl. Beauchamp 1959, Meyer 1901). Die Archianneliden sind größtenteils Mesopsammon-Bewohner ${ }^{* \%}$, die mit Hilfe der ventralen Ciliatur zu einem Gleitkriechen befähigt sind. Vielleicht leitet sich auch Ctenodrilus von Bewohnern des Sandlïckensystems ab; beim Übergang zum Leben zwischen Algen hätte dann die ventrale Bewimperung ihre ursprüngliche Funktion verloren.

Neben dem ventralen Cilienfeld zählt der ausstiilpbare Pharynxbulbus zu den charakteristischen Eigenschaften der Ctenodriliden. Vergleichbare Bildungen finden sich bei vielen Archianneliden (Jaegersten 1947, BeAuchamp 1959, OHM 1963), den Parergodriliden (Karling 1958, Reisinger 1960) sowie nach Dales (1963) bei weiteren Polychaeten-Familien (Pectinariidae, Ampharetidae, Terebellidae u. a.). Die prinzipielle Gleichheit des Baues des Pharynx besteht bei Archianneliden und Ctenodriliden im folgenden: Schlundkopf und Epithelfalten sind hervorstreckbar; die dazu nötige Muskulatur besteht aus einem massiven, im Bogen verlaufenden Sagittalmuskel, der auch die Unterlage des Schlundkopfes bildet, sowie Retraktoren und Haltemuskeln. Eine weitere Übereinstimmung liegt im Bau des eigentlichen Schlundkopfes: Er ist in sich abgeschlossen und besteht aus lamellenartig hintereinander angeordneten Muskelplatten.

Auch die Gattung Potamodrilus (Familie Aeolosomatidae nach Brinkrurst \& Jamieson, Familie Potamodrilidae nach Bunke 1967) besitzt einen ventralen Pharynxbulbus, der jedoch nicht mit dem von Ctenodrilus zu homologisieren ist. Der Schlundkopf ("Zunge“ bei BunkF 1967) zeigt nicht den gleichen anatomischen Bau; ex ist kein in sich abgeschlossenes Muskelpaket; die in ihn hineinziehenden Muskelstränge lassen ein Hervorstrecken aus der Mundöffnung nicht zu.

Brinkhurst (in Brinkhurst \& Jamieson 1971) schließt Potamodrilus und verwandte Gattungen von den Oligochaeten aus; er hält sie für näher mit den Polychaeten verwandt. U. a. verweist er auf das Fehlen eines Clitellums, das das kennzeichnende apomorphe Merkmal der Clitellata ist, sowie auf den ventralen Pharynxbulbus, der einzigartig bei Oligochaeten ist.

Der Bulbus von Potamodrilus läßt sich jedoch zumindest nicht mit dem der Archianneliden, Ctenodriliden (s. oben) und Parergodriliden (vgl. Bunke 1967) homologisieren. Bunke (1967) deutet dies als Zeichen der konvergenten Entstehung eines Pharynxbulbus bei verschiedenen Anneliden, vor allem bei Formen aus dem Lebensraum des Mesopsammons. DALEs (1963) dagegen wertet den Pharynxbulbus als ursprüngliches Annelidenmerkmal, das sich außer bei den Archianneliden auch bei einigen Polychaeten-Familien (s. oben) erhalten hat. Eine genaue Untersuchung dieser Polychaeten wäre wünschenswert.

Eine überzeugende Homologisierung des Pharynxbulbus von Ctenodriliden und

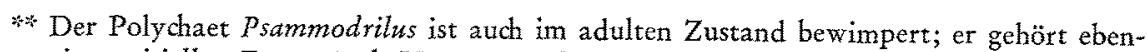
falls zur interstitiellen Fauna (vgl. HartManN-SCHRÖDER 1971). 
Archianneliden fällt schwer, da bisher z. B. keinerlei Angaben über seine Innervierung und nur wenige über seine Entwicklung vorliegen (bei Ctenodriliden: Sokolow 1911). Auch zeigen die Archianneliden eine Reihe von Variationen im Bauplan des Pharynx (Jaegersten 1947, OHM 1963).

Nimmt man eine Homologie des Pharynxbulbus innerhalb der Polychaeten zwischen Archianneliden, Ctenodriliden und einigen anderen Familien an, so kommt den Archianneliden eine Schlüsselrolle bei der Beurteilung des phylogenetischen Werts des Merkmals Pharynxbulbus zu. Sind sie ursprünglich gebaut, sekundär vereinfacht und heterogen oder, wie HERMANs (1969) nach einer sorgfältigen Literatur-Studie annimmt, Polychaeten, die primär an den Lebensraum Mesopsammon angepaßt sind?

Betrachtet man sie als spezialisierte Abkömmlinge einer ancestralen AnnelidenGruppe wie Dales (1963), so ist der Pharynxbulbus ein symplesiomorphes Merkmal, das dann nicht zur Begründung einer näheren Verwandtschaft der einzelnen Archianneliden-Familien herangezogen werden könnte.

Sieht man in den Archianneliden eine heterogene Gruppe (z. B. DoGel 1959, audh Dales 1963), die auf verschiedene Polychaeten-Familien aufzuteilen wäre, so müßte sich bei ihnen und anderen Polychaeten der Bulbus im Hinblick auf Fortbewegung und Nahrungsaufnahme erhalten haben; audh hier wäre eine Urgruppe mit einer derartigen Ausbildung des Pharynx zu fordern.

Nicht auszuschließen ist, daß für diese Formen der Lebensraum Mesopsammon als primär anzusehen ist. Ctenodrilus serratus kommt zwar allein schon wegen seiner Fortbewegungsweise als Sandlïckenbewohner nicht in Betracht, das ventrale Cilienband könnte jedoch primär als Organ für eine derartige ursprüngliche Lebensweise gedient haben.

Elektronenmikroskopische Untersuchungen am Schlundkopf sowie eine Feststellung seiner Innervierung sollen weiteres Material zur Klärung des PharynxbulbusProblems bei Anneliden liefern.

\section{ZUSAMMENFASSUNG}

1. Von dem erstmals für die Fauna von Rovinj (Istrien, Jugoslawien) nachgewiesenen drilomorphen Polychaeten Ctenodrilus serratus (O. SCHMIDT 1857) werden morphologische und biologische Daten mitgeteilt.

2. Der Bau des Schlundkopfes (Pharynxbulbus) läßt einen Vergleich mit dem der Archianneliden zu. Dieser Schlundkopf ist ein Hilfsorgan bei der Nahrungsaufnahme, auch bei der Fortbewegung spielt er eine wichtige Rolle. Auf Grund seines Baues läßt er sich nicht mit dem Pharynxbulbus von Potamodrilus (Klasse Oligochaeta) homologisieren.

3. Dem für die Ctenodriliden charakteristischen ventralen Wimperfeld auf den ersten Segmenten kann keine Funktion für Nahrungsaufnahme und Lokomotion zugesprochen werden.

4. Wimperfeld und farbige Hautdrüsen sowie das Fehlen von mehreren Tentakeln bzw. Tentakelfäden und von Kiemen lassen eine deutliche Abgrenzung zwischen den Familien Cirratulidaes. str. und Ctenodrilidae zu. 
5. Auf Grund der Variationsbreite der Art C. serratus kann C. s. limulicolus SudzukI \& SEKIGUCHI (1972) nicht als valide Unterart betrachtet werden.

Danksagungen. Fräulein L. LANGENSTRASSEN danke ich für zuveriässige technische Assistenz. Herr Prof. Dr. K. Rosenbauer ermöglichte dankenswerterweise die rasterelektronenmikroskopischen Untersuchungen an der Abteilung für Histologie und Embryologie des Anatomischen Instituts der Universität Düsseldorf. Sehr zu Dank für technische Hilfe dabei bin ich Fräulein B. JANSEN verpflichtet.

\section{ZITIERTE LITERATUR}

Beauchamp, P., 1959. Archiannélides. In: Traité de zoologie. Ed. par P. P. Grassé, Masson, Paris 5 (1), 197-223.

Brinkhurst, R. O. \& Jamieson, B. G. M., 1971. Aquatic oligochaeta of the world. Oliver \& Boyd, Edinburgh. 860 pp.

BunKE, D., 1967. Zur Morphologie und Systematik der Aeolosomatidae BEDDARD 1895 und Potamodrilidae now. fam. (Oligochaeta). Zool. Jb. (Syst. Ókol. Geogr. Tiere) 94, 187-368.

Caullert, M. \& Mesntl, F., 1898. Les formes épitoques et l'évolution des Cirratuliens. Annls Univ. Lyon 39, 1-200.

Claparède, A. R. E., 1863. Beobachtungen über Anatomie und Entwicklungsgeschichte wirbelloser Tiere, an der Küste der Normandie angestellt. Engelmann, Leipzig, 120 pp.

Dales, R. Ph., 1963. Annelids. Hutchinson, London, 200 pp.

Dehorne, A, 1924. Multiplication asexuée chez Dodecaceria du Portel par émiettement métamérique, ou processus de cténodrilisation. C. r. hebd. Séanc. Acad. Sci., Paris 178, 123-129.

Dogex, V. A., 1959. Zoology of invertebrates. Sov. Nauka, Moskau, 511 pp.

Fauver, P., 1927. Polychètes sédentaires. Faune Fr. 16, 1-494.

- 1959. Classe des Annélides polychètes. In: Traité de zoologie. Ed. par P. P. Grassì, Masson, Paris 5 (1), 1-196.

Galvagni, E., 1903. Histologie des Genus Ctenodrilus Clap. Arb. zool. Inst. Univ. Wien 15, $46-78$.

Hartmanx-Schröder, G., 1971. Annelida, Borstenwürmer, Polychaeta. Tierwelt Dtl. 58, $1-594$.

Hermans, C. O., 1969. The systematic position of the Archiannelida. Syst. Zool. 18, 85-102.

Jaegersten, G., 1947. On the structure of the pharynx of the Archiannelida with special reference to there-occurring muscle cells of aberrant type. Zool. Bidr. Upps. 25, 551-570.

Karling, T. G., 1958. Zur Kenntnis von Stygocapitella subterranea KnöLlNeR und Parergodrilus beideri ReIsInger (Annelida). Ark. Zool. 11, 307-342.

Katzmann, W., 1972. Die Polychaeten Rovinjs (Istrien/Jugoslawien). Zool. Anz. 188, 116-144.

Kennex, J., 1882. Über Ctenodrilus pardalis Clap. Ein Beitrag zur Kenntnis der Anatomie und Knospung der Anneliden. Arb. zool. zoot. Inst. Würzburg 5, 273-429.

Mesnal, F. \& Caullery, M., 1897. Sur la position systématique du genre Ctenodrilus Cl.AP.: Ses affinités avec les Cirratuliens. C. r. hebd. Séanc. Acad. Sci., Paris 125, 542-544.

Meyer, E., 1901. Studien über den Körperbau der Anneliden. Mitt. zool. Stn Neapel 14, $247-585$.

Monticeldi, F. S., 1893. Sulla Ctenodrilus serratus O. Schmidt. Boll. Soc. Nat. Napoli 7, 39-44.

- 1906. Sessualità e gestazione nel Ctenodrilus serratus $\mathrm{O}$. Sснм. Communicazione preliminare riassuntiva. Atti Congr. Nat. ital. Milano 524-525.

- 1910. Raphidrilus nemasoma MonTrC. Nuovo Ctenodrilide del Golfo di Napoli. Archo zool. ital. 4, 401-436.

OHM, G., 1963. Uber den Bau des Pharynx einiger Archianneliden. Zool. Anz. 171, 179-203.

Peters, W., 1961. Methoden zur Herstellung von Aufhellungspräparaten. Zool. Anz. 167, 233-240. 
- 1968. Vorkommen, Zusammensetzung und Feinstruktur peritrophischer Membranen im Tierreich. Z. Morph. Tiere 62, 9-57.

Rersnger, E., 1960. Die Lösung des Parergodrilus-Problems. Z. Morph. Okol. Tiere 48, $517-544$.

Salvini-Plawen, L. von, 1972. Zur Morphologie und Phylogenie der Mollusken; Die Beziehungen der Caudofoveata und der Solenogastres als Aculifera, als Mollusca und als Spiralia (nebst einem Beitrag zur Phylogenie der coelomatischen Räume). Z. wiss. Zool. 184, 205-394.

SchtarfF, R., 1887. On Ctenodrilus parvulus. Q. J1. microsc. Sci. 27, 591-603.

Schмmрт, O., 1857. Zur Kenntnis der Turbellaria rhabdocoela und einiger anderer Würmer des Mittelmeeres. 2. Beitrag. Sber. Akad. Wiss. Wien 23, 347-366.

Sokolow, I., 1911. Uber eine neue Ctenodrilus-Art und ihre Vermehrung. Z. wiss. Zool, 97, $547-603$.

Stephenson, J., 1930. The Oligochaeta. Clarendon, Oxford. 978 pp.

Sudzuki, M. \& Sекіguchi, K., 1972. Some remarks on five aberrant annelids from the culture water of Japanese Horse-shoe crabs. Scient. Rep. Tokyo Kyoiku Daigaku (B.) 15, 39-56.

VEJDOWSKY, F., 1884. System und Morphologie der Oligochaeten. Rionáč, Prag. 166 pp.

VIERKAus, H., 1971. Ober peritrophische Membranen und andere chitinhaltige Strukturen bei Anneliden, unter besonderer Berücksichtigung von Lumbricus terrestris L. Diss. Univ. Düsseldorf, 99 pp.

WrLfert, M. \& BeCkManN, D., 1972. Ein verbesserter Roto-Kompressor. Mikroskopie 28, 269-273.

Anschrift des Verfassers: Dr. M. Whrfert

Institut für Zoologie

4 Düsseldorf 1

Mettmanner Str. 16

Bundesrepublik Deutschland 